\title{
O Uso de Estratégias de Aprendizagem Cognitivas e Metacognitivas na Disciplina Semipresencial de Processamento da Informação
}

\author{
Denise Goya ${ }^{1}$, Carla Rodriguez ${ }^{1}$, Cláudia A. Araújo ${ }^{1}$, Mirtha L. Fernandez Venero ${ }^{1}$ \\ ${ }^{1}$ Centro de Matemática, Computação e Cognição - Universidade Federal do ABC \\ (UFABC) - Santo André - SP - Brasil \\ \{denise.goya, c.rodriguez, claudia.abrao, mirtha.lina\}@ufabc.edu.br
}

\begin{abstract}
The aim of this paper is to present the results of a research developed with a discipline class of Information Processing, which is offered as blended learning at the Federal University of $A B C$. The focus of the research was to analyze the use of cognitive and metacognitive strategies by the students for programming logic learning and its influence on the performance throughout the course. Using exploratory and descriptive procedures, we present the data collected through an online self-assessment questionnaire and the statistics of accesses to the Virtual Learning Environment. In general, it was possible to identify the most used learning strategies and to extract elements that can be implemented in new resources for the next offers of the discipline in blended learning.
\end{abstract}

Resumo: Este trabalho tem como objetivo apresentar os resultados de uma pesquisa desenvolvida com uma turma de alunos da disciplina Processamento da Informação, cursada na modalidade semipresencial na Universidade Federal do ABC. O foco da pesquisa foi analisar o uso que os alunos fazem de estratégias cognitivas e metacognitivas para a aprendizagem de lógica de programação e sua influência no seu desempenho ao longo da disciplina. Usando procedimentos de caráter exploratório e descritivo, apresentamos os dados coletados por meio de um questionário de autoavaliação online e as estatísticas de acesso ao Ambiente Virtual de Aprendizagem. Em linhas gerais, foi possível identificar as estratégias de aprendizagem mais utilizadas pelos alunos e extrair elementos que podem ser implementados em novos recursos para as próximas ofertas da disciplina na modalidade semipresencial.

\section{Introdução}

O ensino superior de hoje precisa desenvolver nos alunos as habilidades necessárias para resolver problemas complexos, que requerem soluções interdisciplinares. Por isso, a formação interdisciplinar está crescendo a cada dia no Brasil e no mundo. Os cursos interdisciplinares usualmente envolvem técnicas matemáticas, modelagem computacional e aprendizagem baseada em problemas. Assim, esses cursos devem oferecer disciplinas obrigatórias para instrumentalizar o estudante na aplicação de ferramentas computacionais de análise e tratamento de dados e iniciá-lo em linguagens de programação de propósito geral.

A Universidade Federal do ABC é uma das universidades brasileiras com um modelo de ensino superior amplamente interdisciplinar. Conforme estabelece o seu projeto pedagógico, os Bacharelados Interdisciplinares (BI) em Ciência e Tecnologia e em Ciências e Humanidades são as únicas portas de entrada à Instituição para a graduação [UFABC 2015] e formam a base de outros cursos de formação específica.

Esse modelo impõe ao estudante uma grande liberdade, pois as disciplinas não exigem o cumprimento de pré-requisitos. Porém essa liberdade demanda responsabilidade e a mobilização de estratégias de aprendizagem que possam favorecer a aquisição de conhecimentos. Entretanto, nem todos os estudantes chegam ao ensino 
superior de graduação com a mesma maturidade, e uma base de conhecimentos e atitudes apropriada para encarar as habilidades básicas exigidas em um curso interdisciplinar. Um fator complicador desse quadro é que o caráter interdisciplinar da UFABC faz com que as turmas sejam muito heterogêneas, com relação aos perfis dos alunos e suas expectativas profissionais.

Para lidar com a demanda crescente de alunos ingressantes e reprovados nessas disciplinas, a Universidade incentiva a criação de disciplinas para turmas semipresenciais de graduação. A primeira disciplina a ser ofertada de forma semipresencial na UFABC, em 2013, foi Processamento da Informação (PI). A disciplina PI tem por objetivos apresentar os fundamentos sobre manipulação e tratamento da informação, principalmente por meio da explicação e experimentação dos conceitos, e do uso prático da lógica de programação. A ementa de PI cobre conteúdo básico de programação, incluindo: estruturas de seleção e de repetição; vetores e matrizes; métodos/funções. A carga horária é de 60 horas, a ser cumprida em 12 semanas, em regime quadrimestral (Q1, Q2 ou Q3).

Ambas as modalidades, presencial e semipresencial, compartilham a mesma ementa, carga horária e preceito de realização de avaliações teóricas e práticas. E, ambas apresentam índices de reprovação relativamente altos, de mais de $30 \%$. Concretamente, o índice de reprovação na disciplina foi de 33,6\% para as turmas semipresenciais, incluindo reprovação por desempenho e por abandono (média das turmas de 2013.Q1 a 2017.Q1), e 34,1\% para turmas presenciais (média das turmas de 2009.Q3 a 2017.Q1). Esses índices são um pouco maiores, porém não discrepantes, que a média de $30 \%$ de reprovações em disciplinas similares, apurada na Universidade de São Paulo, no período de 2010 a 2014 [Bosse and Gerosa 2015].

Nesse contexto, este artigo tem como objetivo apresentar um recorte da experiência realizada em um recente projeto ${ }^{1}$ desenvolvido na UFABC, que tem como objetivo principal propor materiais que contemplem estratégias de aprendizagem e possam aumentar o engajamento dos alunos na disciplina PI semipresencial. Assim, neste trabalho são apresentadas as estratégias de aprendizagem (cognitivas e metacognitivas) usadas pelos alunos de uma turma de PI semipresencial (2017.Q2) para lidar com o conteúdo sobre raciocínio lógico e para a solução de exercícios de programação. Adicionalmente, o artigo traz uma análise sintética sobre o quanto essas estratégias contribuem para o desempenho dos alunos nessa disciplina.

Além dessa seção introdutória, o artigo está organizado da seguinte forma: a seção 2 descreve a fundamentação teórica usada e apresenta alguns trabalhos relacionados; a seção 3 apresenta a metodologia usada; a seção 4 traz a análise e a discussão dos resultados obtidos; por fim, na seção 5, são abordadas algumas considerações finais.

\section{Estratégias cognitivas e metacognitivas em lógica de programação}

Desde seu surgimento, o ensino semipresencial e a distância tem crescido gradualmente no Brasil e no mundo. Apesar das vantagens que esta modalidade de estudo oferece, a promoção e manutenção da motivação, engajamento e retenção dos estudantes são ainda seus maiores desafios [Fredricks and Mccolskey 2012]. Diversos fatores estão

\footnotetext{
${ }^{1}$ O projeto em questão foi elaborado em resposta ao Edital no ${ }^{\circ}$ 016/2016 de Melhoria do Ensino para propor "trilhas de aprendizagem" diferenciadas para um mesmo conteúdo.
} 
envolvidos nas evasões e reprovações no ensino a distância. Porém, vários estudos têm indicado a importância do uso adequado de estratégias de aprendizagem para o sucesso dos estudantes nesses tipos de cursos [Efklides 2006, Nikolaki and Koutsouba, 2012]. Segundo Silva e Sá (1997), o bom desempenho nas tarefas acadêmicas pode ser alcançado quando o estudante consegue selecionar as estratégias mais adequadas para sua aprendizagem e avaliar seu desempenho durante todo o processo.

As estratégias de aprendizagem podem ser definidas como processos, procedimentos, capacidades complexas e comportamentos empregados para alcançar os objetivos de uma determinada aprendizagem [Flavell 1979, Weinstein and Mayer 1985, Derry and Murphy 1986, Silva and Sá 1997]. Elas podem ser aprendidas de forma intuitiva e natural, ao longo do tempo, ou treinadas para aumentar a efetividade da aprendizagem [Warr and Allan 1998]. De acordo com [Boruchovitch 1999], as estratégias de aprendizagem podem ser divididas em dois grupos: as cognitivas e as metacognitivas. As primeiras referem-se a comportamentos que permitem armazenar as informações mais eficientemente, e.g. elaboração e organização. As estratégias metacognitivas estão relacionadas ao planejamento, monitoramento e regulação dos processos cognitivos. Elas referem-se ao conhecimento e controle sobre a situação atual da atividade cognitiva [Corso et al. 2013].

Vários estudos apontam o julgamento da aprendizagem, o monitoramento das dificuldades e o grau de autoconfiança como fatores importantes para o desempenho em matemática [Garofalo and Lester 1985] e na programação [Shaft 1995]. Por isso, várias iniciativas têm desenvolvido métricas [Gama 2001, Tobias and Everson 2002] e ferramentas para o automonitoramento do aluno para resolver problemas de álgebra e equações lineares [Gama 2001] e lógica de programação [Abreu and Pimentel 2005, Oliveira and Oliveira 2009]. Hadwin e Winne (2001) implementaram uma ferramenta para a autorregulação da aprendizagem, que pode ser usada em qualquer curso. Há trabalhos no contexto brasileiro sobre ambientes para o ensino de programação [Silva et al. 2015]. No entanto, existem poucos resultados que incorporam, em cursos de lógica de programação semipresenciais ou a distância, mecanismos que permitam o estudante reconhecer e autorregular seu processo cognitivo e metacognitivo [Pimentel et al. 2004].

\section{Procedimentos metodológicos}

De acordo com os objetivos propostos para a investigação apresentada neste trabalho, a metodologia de pesquisa científica utilizada tem um caráter exploratório e descritivo [Thiollent 1996]. Assim, recorreu-se a dois métodos de coleta de dados: i) um questionário de autoavaliação online ${ }^{2}$; e ii) a coleta automática de dados de acesso dos alunos aos materiais disponíveis no Ambiente Virtual de Aprendizagem (AVA) TIDIA4 [Beder et al. 2007].

O questionário de autoavaliação online foi aplicado de forma a manter o anonimato do aluno e contou com três seções complementares. A primeira seção, Dados Gerais, permite identificar o aluno de acordo com a faixa etária, sexo, o ano de ingresso na universidade, o turno em que estuda, se trabalha etc. A segunda seção, Sobre a Prova, buscou investigar a expectativa que o aluno tinha em relação ao seu desempenho na primeira prova realizada e, se cometeu erros, quais os prováveis motivos (falta de atenção; falta de estudo; má administração do tempo; nervosismo) que o levaram a

\footnotetext{
${ }^{2}$ Questionário disponível em https://goo.gl/Cn3PNf
} 
cometê-los. Por fim, a terceira seção, Sobre o seu desempenho na disciplina PI, foi composta por questões fechadas, de orientação mais geral, por exemplo, o nível de desempenho (excelente, bom, satisfatório, ruim, péssimo), que julgavam ter até o momento, e a qual elemento (conhecimento anterior sobre o conteúdo; engajamento nas atividades; participação; qualidade do material), em menor ou maior grau, estava relacionado o nível de desempenho atribuído.

Além destas questões gerais, a terceira seção contou com questões mais específicas, sobre os hábitos de estudo adotados pelo aluno até a realização da prova. Em relação às questões mais específicas, de forma similar ao trabalho de [Alliprandini et al. 2014], optou-se por adaptar e incluir no questionário itens relacionados às estratégias de aprendizagem cognitivas e metacognitivas mobilizadas pelos alunos. Assim, um conjunto de 33 afirmações, adaptadas dos 32 itens referentes às estratégias de aprendizagem metacognitivas positivas e negativas (ou disfuncionais), e dos 19 itens que caracterizam as estratégias de aprendizagem cognitivas, propostos por Santos e Boruchovitch (2008), foram incluídas no questionário de autoavaliação (todos os itens constam nas figuras 1 e 2). As respostas às afirmações seguiam o formato "múltipla escolha", para serem respondidas de acordo com a frequência que o aluno considerasse mais próxima da sua situação: Sempre, Às Vezes, Raramente e Nunca.

O questionário de autoavaliação foi aplicado após a realização da primeira prova (P1), cujo conteúdo abrangeu estruturas básicas de programação, como seleção e laços de repetição, sem incluir vetores e funções, que foram tópicos posteriores à P1. Somente realizaram a P1 os alunos que não haviam efetuado o cancelamento de matrícula, opção que poderia ser assumida após a aula inaugural presencial e obrigatória e após duas semanas de atividades. Assim, não participaram da autoavaliação os alunos que já haviam reconhecido dificuldades com a dinâmica das aulas na modalidade semipresencial e/ou com as regras estabelecidas na aula inaugural.

Além do questionário de autoavaliação, uma análise das interações dos alunos com o AVA foi realizada. Os dados das interações foram extraídos por meio da ferramenta Estatísticas, que possibilita a elaboração de relatórios de acesso dos alunos aos vários recursos disponíveis, como a quantidade de vezes que os alunos acessaram o ambiente e materiais específicos.

A análise dos dados coletados por meio dos métodos e instrumentos apresentados priorizou o tratamento quantitativo, articulado com uma dimensão qualitativa, o que possibilitou uma interpretação mais significativa sobre o fenômeno estudado. No próximo tópico, apresentamos os resultados alcançados, bem como a análise e discussão dos aspectos mais significativos da pesquisa.

\section{Resultados: análise e discussão}

O questionário foi respondido por 133 alunos e revelou, principalmente, que os alunos participantes da pesquisa afirmam adotar com mais frequência (Sempre) em seu percurso de aprendizagem estratégias metacognitivas (Figura 1) do que estratégias cognitivas (Figura 2).

É possível observar que as estratégias metacognitivas apresentadas na Figura 1 são todas positivas e importantes para o processo de aprendizagem [Santos and Boruchovitch 2008], porém algumas delas são aplicadas Raramente ou Nunca em proporções relativamente altas. 
VI Congresso Brasileiro de Informática na Educação (CBIE 2017)

Anais dos Workshops do VI Congresso Brasileiro de Informática na Educação (WCBIE 2017)

A estratégia menos usada (a de número 20, Figura 1) mostra que os alunos pouco se reúnem para estudar juntos. Nesse sentido, propor mais atividades em grupo pode estimular os alunos a aumentarem esse tipo de interação e, eventualmente, promover reflexos positivos em seu aprendizado.

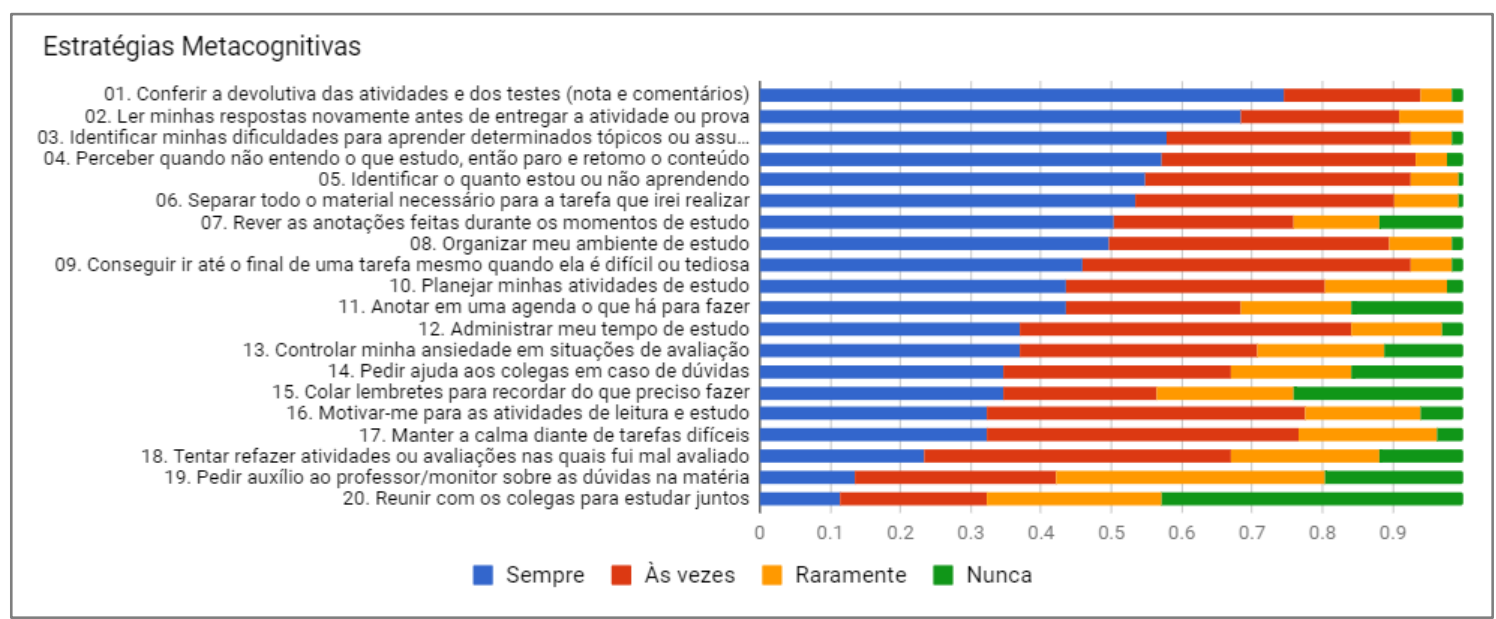

Figura 1 - Estratégias metacognitivas adotadas pelos alunos ${ }^{3}$

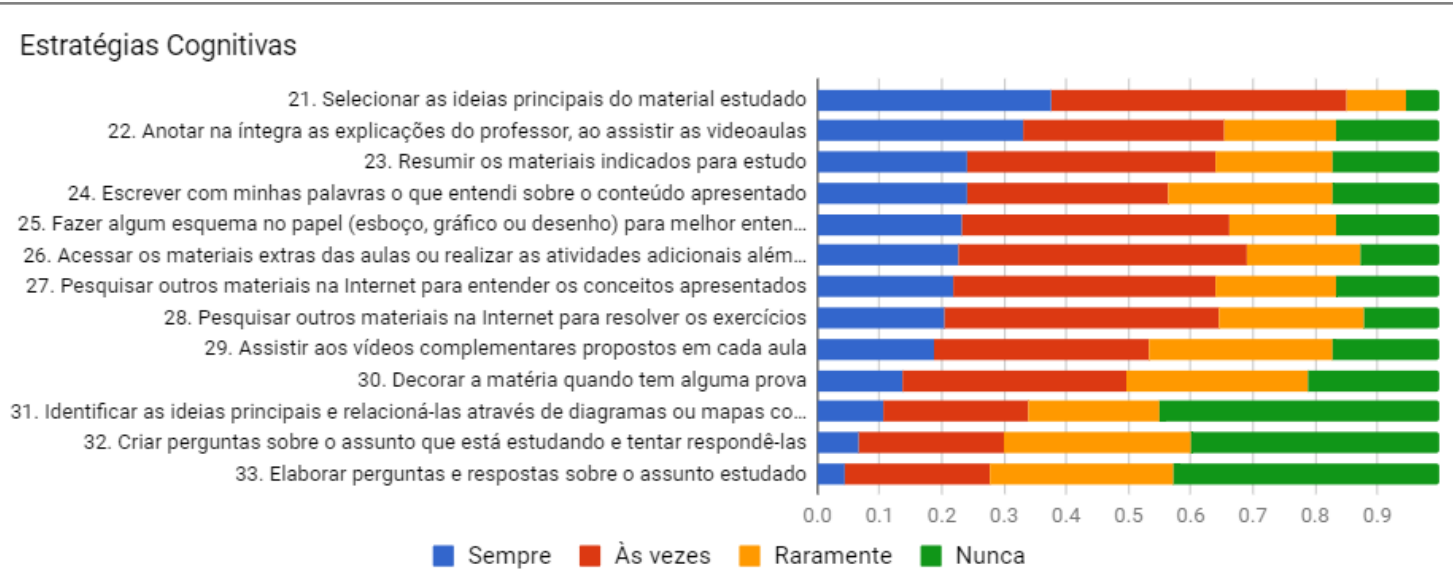

Figura 2 - Estratégias cognitivas adotadas pelos alunos

A estratégia 19 (Figura 1), como uma das menos adotadas é, de certa forma, preocupante. No caso em particular analisado, que envolve alunos da disciplina semipresencial PI, cada aluno tem à disposição um professor e um monitor para resolução de dúvidas na matéria, além de ser amplamente enfatizado e incentivado que o aluno interaja com seus professores e monitores, como uma forma de suprir o pouco contato presencial. Uma possível razão para a pouca adoção da estratégia metacognitiva 19 (Figura 1) ocorrer é que a interação é predominantemente assíncrona e por meio de troca de mensagens escritas, o que demanda certo tempo (tipicamente inferior a $36 \mathrm{~h}$, e limitado a 72h) de espera pela resposta, e os alunos preferem buscar respostas imediatas para suas dúvidas usando a Internet (ver estratégias cognitivas 27 e 28, Figura 2).

Em atenção à estratégia metacognitiva 18 (Figura 1), Tentar refazer atividades ou avaliações nas quais fui mal avaliado, é possível, por exemplo, acrescentar tarefas ao conjunto de atividades propostas que recompensem o aluno que fizer novamente as

\footnotetext{
${ }^{3}$ Para melhor legibilidade, as estratégias cognitivas e metacognitivas são listadas em: https://goo.gl/yGgQAb
} 
VI Congresso Brasileiro de Informática na Educação (CBIE 2017)

Anais dos Workshops do VI Congresso Brasileiro de Informática na Educação (WCBIE 2017)

atividades nas quais tenha alcançado baixo desempenho. Isso pode estimular o aluno a aprender com seus próprios erros e/ou permitir que o docente perceba quando o aluno repete os mesmos erros ou fornece indícios de que não assimilou o conteúdo.

As estratégias metacognitivas 06 a 17 (Figura 1) estão relacionadas à organização dos estudos e controle emocional, aspectos esses que podem ser explorados pelos docentes para incentivar que os alunos melhor planejem e administrem seu estudo (vital na modalidade semipresencial), e possam identificar que mudanças comportamentais seriam positivas para um melhor aproveitamento. A inclusão de checklists nas atividades é um exemplo de prática que pode auxiliar nesses dois tópicos, de organização e de controle da ansiedade e nervosismo.

As estratégias metacognitivas 03 a 05 (Figura 1) revelam que mais de $40 \%$ dos alunos nem sempre conseguem identificar suas dificuldades, ou mensurar o quanto estão aprendendo. Possivelmente, alguns desses alunos são os mesmos que se encontram entre os $26 \%$ que nem sempre seguem a estratégia 01 , de conferir a devolutiva com nota e comentários fornecida individualmente aos alunos a cada atividade. Esses resultados também estão associados a outros, como o de que mais de $90 \%$ dos alunos não comparecem à vista presencial de provas junto a seus professores e de que $13 \%$ afirmam não ter lido a solução da prova (conforme apurado em outro questionário anonimizado).

Em trabalho recente, Alliprandini et al. (2014) ressaltam a necessidade das estratégias cognitivas e metacognitivas ocorrerem de forma interligada para que se atinja a aprendizagem. Entretanto, comparando-se as Figuras 1 e 2, é notável que os alunos usam menos as estratégias cognitivas (Figura 2).

As estratégias cognitivas menos usadas, 32 e 33 (Figura 2), requerem que o aluno tome a iniciativa de refletir sobre o assunto estudado. Tais estratégias podem ser instigadas, por exemplo, no próprio material oferecido ou nos enunciados das atividades. A terceira estratégia menos usada, a de número 31, é uma evolução das estratégias 21 a 25, que são as mais usadas. Isto é, os alunos selecionam as ideias principais, resumem, escrevem com as próprias palavras sua compreensão sobre o assunto, e chegam a fazer esquemas (mais de $30 \%$ dos alunos Sempre fazem isso, e mais de $70 \%$ o fazem ao menos Às vezes). No entanto mais de $25 \%$ Nunca chegam a relacionar os conceitos em diagramas ou mapas mais complexos, e perto de $55 \%$ o fazem Raramente ou Nunca, conforme apresentado na Figura 2. Idealmente, o aluno deveria explorar mais as estratégias 26 e 29, de acessar os materiais e vídeos adicionais (disponíveis no ambiente), além de realizar as atividades não obrigatórias. Entretanto, o aluno prefere pesquisar outros materiais na Internet (estratégias 27 e 28) a assistir às videoaulas complementares já oferecidas (estratégia 29).

Em respostas apuradas no questionário de autoavaliação, aplicado após a primeira prova escrita, a expectativa dos alunos foi compatível com o conceito efetivamente obtido (Figura 3). Esse resultado demonstra que os alunos, em geral, possuem boa percepção do próprio aprendizado e de suas dificuldades com relação ao conteúdo inicial da disciplina. Na Figura 3, conceito "A" equivale a desempenho excelente, enquanto " $D$ " aponta aproveitamento deficiente, porém significa aprovação; conceito " $F$ " representa reprovação. Esse desempenho foi condizente com a autoavaliação global (54\% dos alunos disseram estar com desempenho em PI, de forma geral, Excelente ou Bom). Isso pode estar relacionado com o engajamento, qualidade do material e conhecimento prévio, conforme relatado pelos próprios alunos (Figura 4). 
VI Congresso Brasileiro de Informática na Educação (CBIE 2017)

Anais dos Workshops do VI Congresso Brasileiro de Informática na Educação (WCBIE 2017)

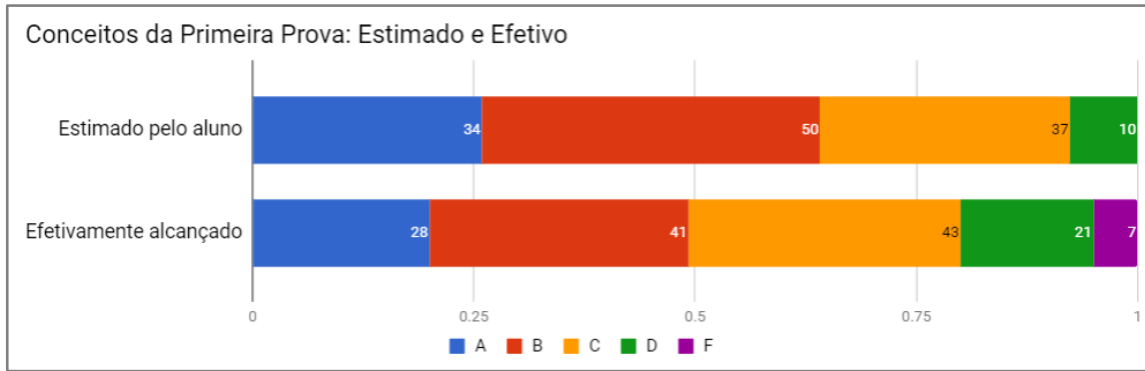

Figura 3 - Distribuição de conceitos da P1: estimados pelos alunos e efetivos

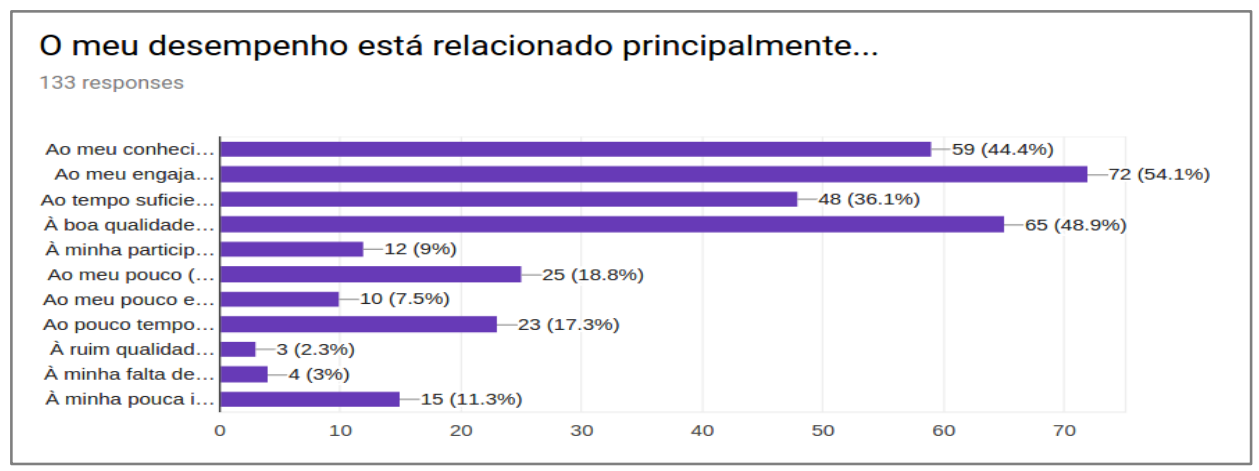

Figura 4 - Autoavaliação global sobre o desempenho na disciplina PI

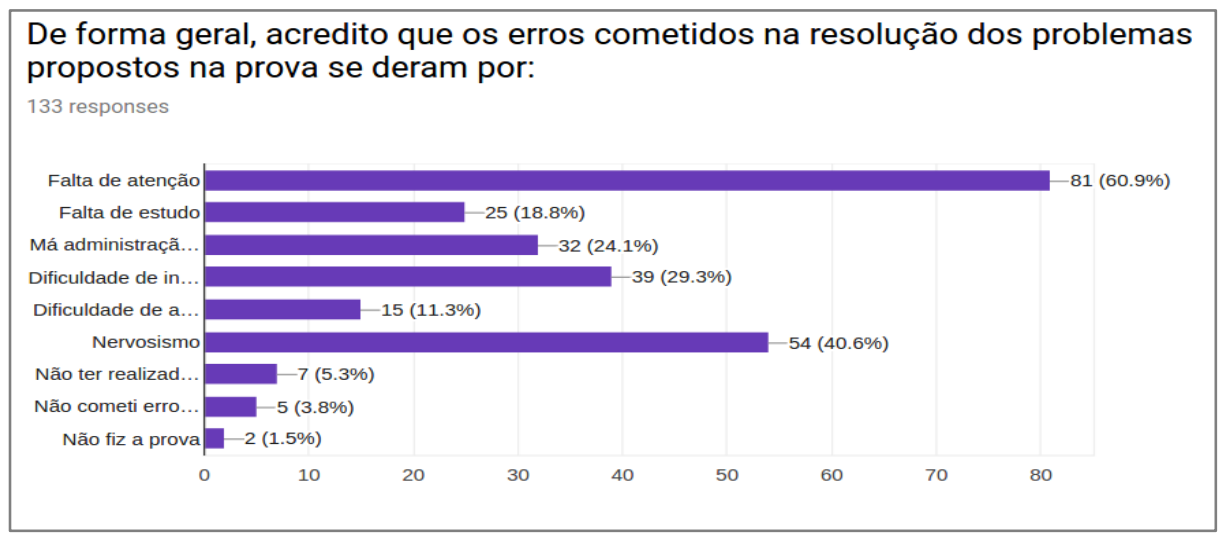

Figura 5 - Aspectos considerados pelos alunos sobre os erros cometidos na P1

No caso da P1, os erros cometidos na prova, de acordo com os alunos, foram devidos principalmente à falta de atenção, ao nervosismo e à dificuldade de interpretação dos enunciados dos problemas (Figura 5).

A partir de dados coletados no AVA, foi possível observar que os 21 alunos com maior frequência neste ambiente foram os que apresentaram melhor desempenho na P1, concentrando proporcionalmente mais conceitos A e B, quando comparados com a turma inteira (Figura 6). No entanto também reúne proporção um pouco maior de $\mathrm{F}$ ( $10 \%$ entre os mais frequentes, contra $5 \%$ de $\mathrm{F}$ na turma toda).

Foi realizado o monitoramento dos acessos a recursos complementares, que não fazem parte dos materiais primários de cada aula, como o são as videoaulas e os slides, no caso de PI. Figuram como recursos adicionais, infográficos, animações e resumos, e apenas uma parcela dos alunos fizeram acessos frequentes a esses materiais. Foi possível detectar que apenas 21 alunos (11,2\% dos matriculados) fizeram uso 
VI Congresso Brasileiro de Informática na Educação (CBIE 2017)

Anais dos Workshops do VI Congresso Brasileiro de Informática na Educação (WCBIE 2017)

sistemático desse material adicional. Comparando o desempenho desses 21 alunos na P1 com o desempenho da turma, observa-se que houve proporcionalmente mais A e mais $\mathrm{F}$ no grupo de alunos que acessaram os recursos complementares. Esse resultado coincide com o de alunos Mais frequentes, que mais acessaram o AVA (Figura 6).

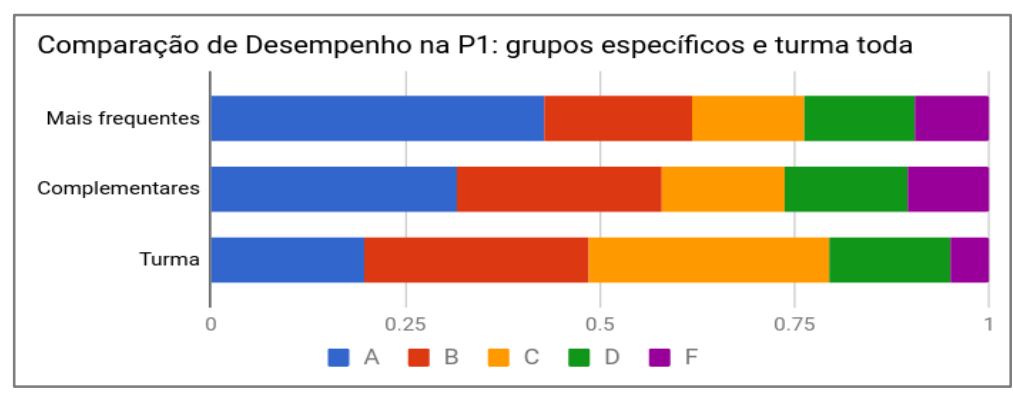

Figura 6 - Comparação de desempenho obtido na primeira avaliação entre os alunos que mais visitaram o AVA, o grupo que acessou materiais complementares e a turma inteira

Uma possível conclusão para essa coincidência é que muitos dos alunos que mais exploraram os materiais extras disponíveis no ambiente de aprendizagem são aplicados, fazem uso das estratégias de aprendizagem e alcançam bons conceitos. Entretanto, uma parcela (10\% do grupo) acessa frequentemente os materiais complementares por sentir dificuldades de aprendizado, mas não consegue converter o esforço em conhecimento do conteúdo e, portanto, em bom conceito na avaliação. Analogamente, os alunos que mais visitaram o ambiente também se dividem em dois grupos: os que alcançam bom desempenho (maioria) e os que não conseguem obter bom aproveitamento (também 10\% do grupo), apesar dos muitos acessos aos materiais.

\section{Considerações finais}

A motivação para realização deste trabalho deriva da compreensão que o uso de estratégias cognitivas e metacognitivas, e recursos diversificados, para apoiar a aprendizagem dos conteúdos da disciplina PI, podem trazer resultados positivos para os alunos. Assim, o objetivo deste artigo foi apresentar as estratégias de aprendizagem (cognitivas e metacognitivas) usadas pelos alunos de uma turma de PI semipresencial (2017.Q2), além de uma análise sintética sobre o quanto essas estratégias contribuíram para o desempenho dos alunos na disciplina.

Conforme apresentado, foi possível identificar que os alunos da turma semipresencial 2017.Q2 utilizaram mais as estratégias metacognitivas para aprendizado dos conteúdos da disciplina de PI. Através da análise das informações extraídas dos instrumentos de coleta de dados usados, foi possível identificar também que alguns alunos se beneficiaram mais que outros dos materiais extras oferecidos. Estes resultados estão alinhados com as diretrizes de pesquisa e trabalhos futuros. Por exemplo, acreditase que, para serem efetivos, os recursos e estratégias cognitivas e metacognitivas a serem aplicados deverão ser baseados no estabelecimento de "trilhas de aprendizagem" diferenciadas para um mesmo conteúdo. Dessa forma, como conclusão deste trabalho, é possível considerar que, no caso da disciplina PI semipresencial, o foco deve estar no uso de recursos que contemplem estratégias de aprendizagem cognitivas e metacognitivas capazes de promover-a motivação e o engajamento e possam ampliar o desempenho dos alunos no aprendizado dos conteúdos propostos. Dentre os recursos a serem utilizados, prioriza-se, por exemplo, o uso de objetos de aprendizagem como 
resumos, infográficos, animações, jogos digitais etc, aos quais é viável incluir roteiros que auxiliem os alunos no planejamento, avaliação e regulação da aprendizagem, como elementos que ampliem a capacidade de abstração, direcionem o raciocínio para o reconhecimento de detalhes e ofereçam oportunidades para reflexão sobre como organizar o pensamento.

Em síntese, espera-se que esse trabalho inicial, exploratório e descritivo sobre um contexto particular possa ampliar as reflexões sobre o desenvolvimento dos materiais que apoiarão as próximas ofertas de PI semipresencial de forma a reduzir o percentual de reprovação e abandono na disciplina. Nesse sentido, em trabalhos futuros, a avaliação dos resultados se centrará em verificar se houve um maior aproveitamento das turmas envolvidas na utilização desta metodologia em relação à aprendizagem do conteúdo, e uma diminuição das taxas de desistência e reprovação, em comparação com os resultados obtidos nas ofertas anteriores. Espera-se ainda que os resultados alcançados, principalmente no que diz respeito à incorporação das estratégias cognitivas e metacognitivas aos recursos utilizados, possam ser significativos não só para PI como para outras disciplinas oferecidas na modalidade semipresencial.

\section{Referências}

Abreu, C. D. O. and Pimentel, E. P. (2005). Recursos avançados de demonstração de resultados de um ambiente de avaliação de habilidades metacognitivas em lógica de programação. In XI Congreso Argentino de Ciencias de la Computación.

Alliprandini, P. M. Z.; Schiavoni, A.; Méllo, D.de and Sekitani, J.T. (2014). Estratégias de aprendizagem utilizadas por estudantes na educação a distância: implicações educacionais. Psicologia da Educação, (38), 05-16.

Beder, D.; Silva, A.; Otsuka, J.; Silva, C. and Rocha, H. (2007). A Case Study of the Development of e-Learning Systems Following a Component-based Layered Architecture. ICALT 2007: 21-25

Bosse, Y. and Gerosa, M. A. (2015). As Disciplinas de Introdução à Programação na USP: um Estudo Preliminar. In: Anais dos Workshops do Congresso Brasileiro de Informática na Educação. 2015. p. 1389.

Boruchovitch, E. (1999). Estratégias de aprendizagem e desempenho escolar: considerações para a prática educacional. Psicologia: Reflexão e Crítica, 12, 361-376.

Corso, H. V.; Sperb, T. M.; Jou, G. I. and Salles, J. F. (2013). Metacognição e funções executivas. Relações entre os conceitos e implicações para a aprendizagem. Psicologia: teoria e pesquisa, 29, 21-29.

Derry, S. J. and Murphy, D. A. (1986). Designing systems that train learning ability: From theory to practice. Review of Educational Research, 56, 1-39.

Efklides, A. (2006) Metacognition and affect: What can metacognitive experiences tell us about the learning process?, Educational Research Review, 1(1): 3-14.

Flavell, J. H. (1979). Metacognition and cognitive monitoring: A new area of cognitive developmental inquiry. American Psychologist, 34, 906-911. 
VI Congresso Brasileiro de Informática na Educação (CBIE 2017)

Anais dos Workshops do VI Congresso Brasileiro de Informática na Educação (WCBIE 2017)

Fredricks, J. and Mccolskey, W. (2012). The Measurement of Student Engagement: A Comparative Analysis of Various Methods and Student Self-report Instruments. In Handbook of Research on Student Engagement, Springer-Verlag, New York.

Gama, C. (2001). Metacognition and reflection in ITS: increasing awareness to improve learning. In J. D.Moore (ed.), Proceedings of the Artificial Intelligence in Education Conference, pp. 492-495, Montreal: CA. IOS Press.

Garofalo, J. and Lester, F. (1985). Metacognition, Cognitive Monitoring, and Mathematical Performance. Journal for Research in Mathematics Education, 16(3): 163-176.

Hadwin, A.F. and Winne, P. H.(2001). CoNoteS2: A software Tool for Promoting SelfRegulation. Educational Research and Evolution, 7(2-3), 313-334.

Nikolaki, E. and Koutsouba, M. I. (2012). Support and promotion of self-regulated learning through the educational material at the Hellenic Open University. Turkish Online Journal of Distance Education, 13, 226-238.

Oliveira, M. G. and Oliveira, E. (2009) Avaliações Metacognitivas Online para Nivelamento de Alunos. In: 15o. Congresso Internacional ABED de Educação a Distância, 2009, Fortaleza, CE. Anais do 15o. CIAED, 1-10.

Pimentel, E. P. and Omar, N.; Mustaro, P.N. and França, V.F. (2004) Um modelo para avaliação e acompanhamento contínuo da aprendizagem. In: XV SBIE - Simpósio Brasileiro de Informática na Educação, SBC.

Santos, A. A. A., and Boruchovitch, E. (2008). Escala de estratégias de aprendizagem para Universitários, Manuscrito não publicado, Unicamp, Campinas, Brasil.

Shaft, T. M. (1995). Helping programmers understand computer programs: the use of metacognition. DATA BASE Advances, 26(4), 25-46.

Silva, A. L. and Sá, I. (1997). Saber estudar e estudar para saber. Porto Editora.

Silva, P., Fechine, J. and Costa, E. (2015). Um Mapeamento Sistemático sobre Iniciativas Brasileiras em Ambientes de Ensino de Programação. Anais do XXVI Simpósio Brasileiro de Informática na Educação, p. 367 - 375.

Thiollent, M. (1996). Metodologia da pesquisa - ação. 2. ed. São Paulo: Cortez.

Tobias, S. and Everson, H. T. (2002). Knowing what you know and what you don't: further research on metacognitive knowledge monitoring. College Board Research Report 2002-3, College Entrance Examination Board: New York.

UFABC (2015). Projeto Pedagógico do Curso Bacharelado em Ciência e Tecnologia. Disponível em http://antigo.ufabc.edu.br/images/stories/pdfs/administracao/ConsEP/ anexo-resolucao-188-revisao-do-ppc-bct-2015.pdf. Acesso em agosto de 2017.

Warr, P. and Allan, C. (1998). Learning strategies and occupational training. International Review of Industrial and Organizational Psychology, 13, 83-121.

Weinstein, C. E. and Mayer, R. E. (1986). The teaching of learning strategies. In M. Wittrock (Ed.). Handbook of research on teaching (pp. 315-327). Nova York: Macmillan. 